\title{
Unexpected location of endometrial adenocarcinoma - a case report
}

\author{
Mircea-Octavian Poenaru ${ }^{1,2)}$, Liana Pleş ${ }^{1,2)}$, Romina-Marina Sima ${ }^{1,2)}$, Petronela Ionescu33), \\ COSMIN FlORESCU ${ }^{4}$, ILINCA NEACŞU ${ }^{5,6)}$, CĂTĂLINA DianA STĂNICĂ ${ }^{1,2)}$, AdRIAN NEACŞU ${ }^{1,2)}$ \\ 1) Department of Obstetrics and Gynecology, Carol Davila University of Medicine and Pharmacy, Bucharest, Romania \\ 2) Department of Obstetrics and Gynecology, Bucur Maternity, St. John Clinical Hospital, Bucharest, Romania \\ 3) Department of Pathology, Dr. Victor Babeş Infectious and Tropical Diseases Clinical Hospital, Bucharest, Romania \\ 4) National Center of Pathology, Laboratoire National de Santé, Luxembourg \\ 5) Department of Pathology, Binafarm, Bucharest, Romania \\ 6) Translational School of Biological Sciences, Faculty of Biology, Medicine and Health, The University of Manchester, \\ Manchester, UK
}

\begin{abstract}
Endometrial adenocarcinoma (ADK) is one of the most common uterine cancer and the fourth neoplasia mortality cause in women according to the literature data. ADK is encountered in the sixth decade of life, the mean age being 63 years. Only $2-5 \%$ of cases are found in women less than 40 years old. We present the case of a 63-year-old woman admitted in our Clinic for hypogastric pain and reduced vaginal sanguinolent discharge. The genital examination and transvaginal ultrasound (US) scan were strongly suggestive for a type 0 myoma tending to be expelled through the cervical canal. Laparotomy and total hysterectomy with bilateral adnexectomy were performed. Histological examination identified a grade G1 well-differentiated endometrioid ADK without uterine wall invasion. The immunohistochemistry study for estrogen receptors expression revealed moderate and strong nuclear immunostaining in more than $70 \%$ of the tumoral cells, as well as a mosaic p16 immunoexpression, a cytokeratin 7 (CK7) immunophenotype, no p53 overexpression and low Ki67 index (estimated at 10-15\%). Considering the incidental finding, the early stage and limited localization of the ADK at the myoma surface level surgery was considered sufficient as treatment but follow-up was conducted by magnetic resonance imaging (MRI) and general examination every six months. The most interesting aspect of the case was the atypical presence of an active myoma in menopause and the totally unsuspicious US appearance of the endometrium. The endometrial ADK was accidentally discovered in an unusual specimen without any other symptom.
\end{abstract}

Keywords: endometrial adenocarcinoma, endometrium, myoma, Ki67 immunoexpression.

\section{a Introduction}

Endometrial adenocarcinoma (ADK) is one of the most common uterine cancer and the fourth neoplasia mortality cause in women according to the literature data [1]. ADK is encountered in the sixth decade of life, the mean age being 63 years. Only $2-5 \%$ of cases are found in women less than 40 years old [2]. The etiology is still unclear, but many studies endorse the natural or iatrogenic hyperestrogenism without progesterone adequate secretion, as a potential cause. The same studies suggest that combined estroprogestative pills can reduce the risk of endometrial neoplasia both before and after menopause [3].

\section{Aim}

By this case report, we aim to underline the importance of carefully investigation of apparent benign uterine condition in postmenopause, since it can hide a more serious pathology.

\section{$\square$ Case presentation}

We present the case of a 63 -year-old woman admitted in our Clinic for colicative hypogastric pain and reduced bloody vaginal discharge. The symptoms occurred in the last week and the pain was gradually increasing in severity. The patient's history revealed two vaginal births and three abortions, menopause for 12 years, mild essential high blood pressure, and type II diabetes. General examination indicated a moderate obesity [body mass index (BMI) of $32.3 \mathrm{~kg} / \mathrm{m}^{2}$ and ambulatory blood pressure (ABP) of $170 / 87 \mathrm{mmHg}]$. At the genital examination, a small amount of brownish blood was found in the vagina, the cervical ostium was dilated and occupied by a tumoral structure covered with necrotic debris and bleeding when touched. The uterus was slightly enlarged, of firm consistency and very sensitive no adnexal mass was found. We performed transvaginal ultrasound (US) scan that showed the slightly enlarged uterus, occupied by a tumor-like hypoechoic structure that protruded in the cervical canal with a length of $86 \mathrm{~mm}$ and large implantation base), well delimitated, without significant Doppler signal and without relationship with the thin, atrophic surrounding endometrium. The US aspect was strongly suggestive for a type 0 myoma, tending to be expelled through the cervical canal. Considering the myoma size and its large attachment at the uterine

This is an open-access article distributed under the terms of a Creative Commons Attribution-NonCommercial-ShareAlike 4.0 International Public License, which permits unrestricted use, adaptation, distribution and reproduction in any medium, non-commercially, provided the new creations are licensed under identical terms as the original work and the original work is properly cited. 
fundus, we did not attempt to excise it by torsion and decided in favor of laparotomy and total hysterectomy with bilateral adnexectomy was performed.

Patient's informed consent was obtained for treatment and data research and study according to the Hospital's protocols.

Grossing of the specimen showed a mass occupying the uterine cavity, measuring $82 \times 53 \mathrm{~mm}$, attached at the uterine fundus and protruding through the cervical ostium. On cross section, the tumor has a yellowish appearance, was of reduced consistency and showed areas of necrosis (Figure 1, A and B). The rest of the endometrial cavity was lined by a pale atrophic mucosa, without any other suspicious areas of associated neoplasia. Several representative sections were submitted for histopathological (HP) exam.

Microscopic examination of slides showed, from the architectural point of view, an extensive and confluent glandular epithelial growth. The proliferation was made of back-to-back endometrial glands, with little or no intervening stroma with cribriform, microacinar or papillary configurations; in some areas, true papillae were observed. At the cytological level, the regular oval nuclei with finely distributed chromatin and rare mitotic figures placed the tumor in nuclear grade 1 category. The low and highpower HP changes allowed the differential diagnosis with endometrial clear cell and serous carcinoma.

The final diagnosis was well-differentiated endometrioid

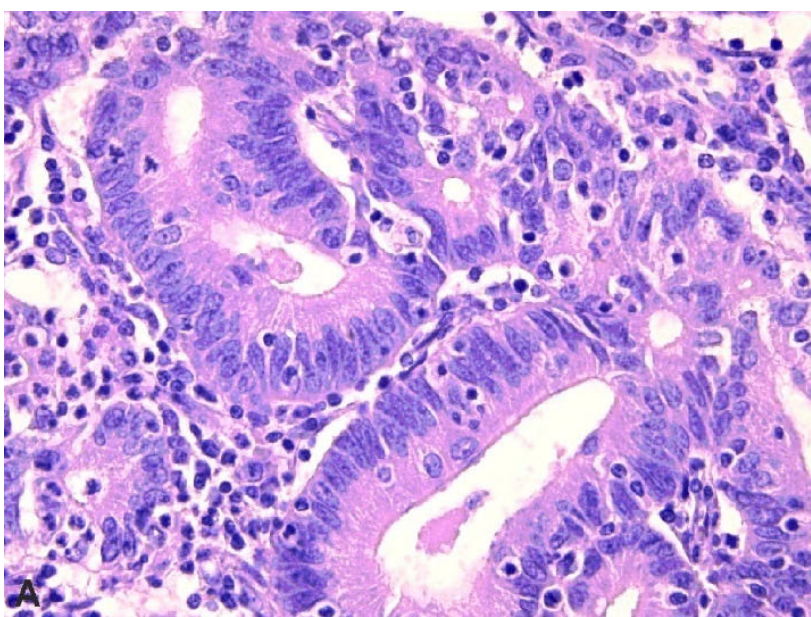

ADK, histological grade 1 (G1) confined to endometrium covering the myoma, without myometrial invasion (Figure 2, A and B).
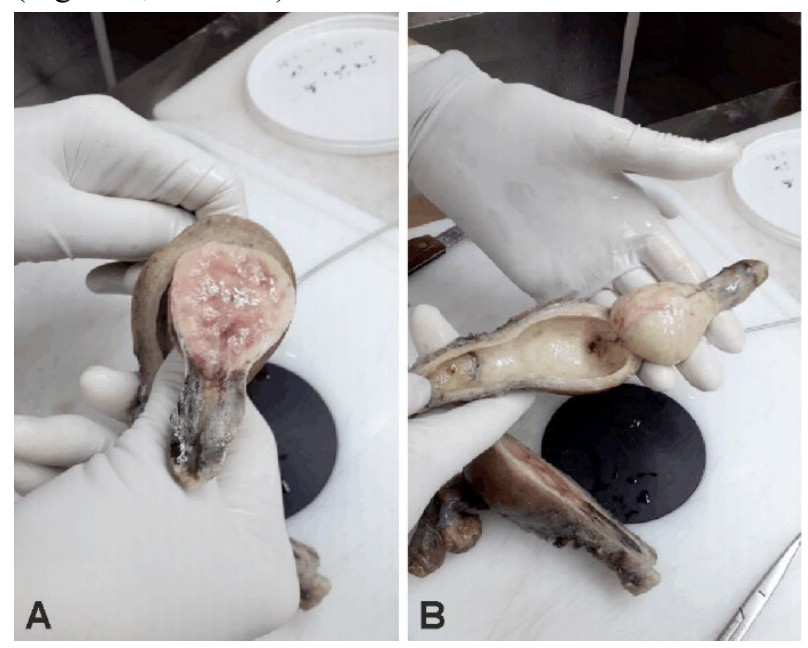

Figure 1 - (A and B) Macroscopic aspect of the hysterectomy specimen opened in longitudinal section along the lateral margins of the uterus. There was an easily detachable polyploid pedunculated mass, only focally attached to the uterine fundus wall by a fine stalk. On cross section, an extensive necrotic process was identified in the central portions and at the level of the intracervical part. Uterine mucosa looks pale and atrophic without any suspect or hemorrhagic areas.

Figure 2 - (A and B) Well-differentiated endometrioid adenocarcinoma without myometrium wall invasion. Histological architecture: extensive, complex and confluent back-to-back epithelial glandular growth pattern, in which individual endometrial glands, with little or no intervening stroma, merge and show budding and branching phenomena, focally creating papillary and pseudo-papillary structures $(B)$. Cytological features: regular oval nuclei of similar shape and size, rare mitoses and focally mild atypia (A). Hematoxylin-Eosin $(H E)$ staining: $(A) \times 400 ;(B) \times 100$.

According to the protocols, in order to provide an appropriate management of the case, immunohistochemistry (IHC) tests were carried out on the formalin-fixed paraffinembedded tumor samples. The examination revealed moderate and strong nuclear immunostaining for estrogen receptors (ERs) in more than $70 \%$ of the tumor cells (Figure 3). We assessed as well p16 and p53 immunoexpression. A focal p16 immunoexpression was found (Figure 4). The p53 immunoreaction came out patchy and weak in scattered nuclei (Figure 5). As expected, an intense membranous cytokeratin 7 (CK7) immunopositivity was identified (Figure 6). The Ki67 labeling index recorded as percentage of tumoral nuclear immunostaining revealed a low proliferation index estimated at $10-15 \%$. Considering the incidental finding, the early stage and limited localization of ADK at the myoma level, conservative surgery was considered sufficient as treatment. Follow-up was conducted by magnetic resonance imaging (MRI) and general examination and US every six months with normal results.

\section{a Discussions}

Abnormal uterine bleeding is the most common symptom in case of endometrial neoplasia [4]. In a recent study, Desai et al. stated that almost $90 \%$ of the endometrial ADKs are symptomatic and revealed by abnormal uterine bleeding mainly in menopause [5]. 
There are also cases that are incidentally discovered on hysterectomy specimens performed for benign conditions without positive previous histology. In $1 \%$ of the specimens, uterine neoplasia was discovered and about $75 \%$ of them are represented by ADK. The same study indicates that occult ADK risk is about $4.4 \%$ for women after 75 years old that underwent hysterectomy for apparently benign

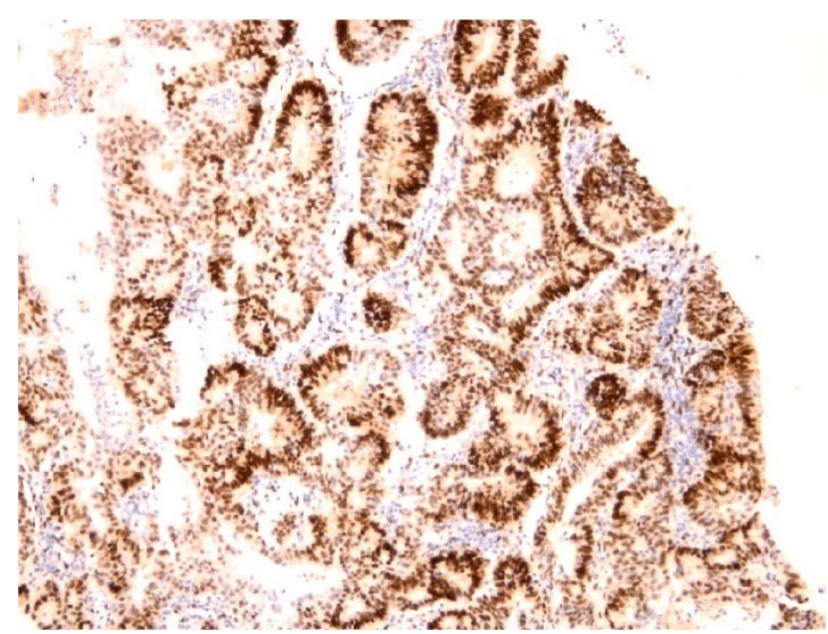

Figure 3 - ERs were assessed by immunohistochemistry, evaluating the percentage of positive cells and intensity of nuclear immunostaining. There was moderate and strong nuclear immunostaining for ERs in more than 70\% of the tumor cells. Anti-ER antibody immunostaining, $\times 100$. ERs: Estrogen receptors.

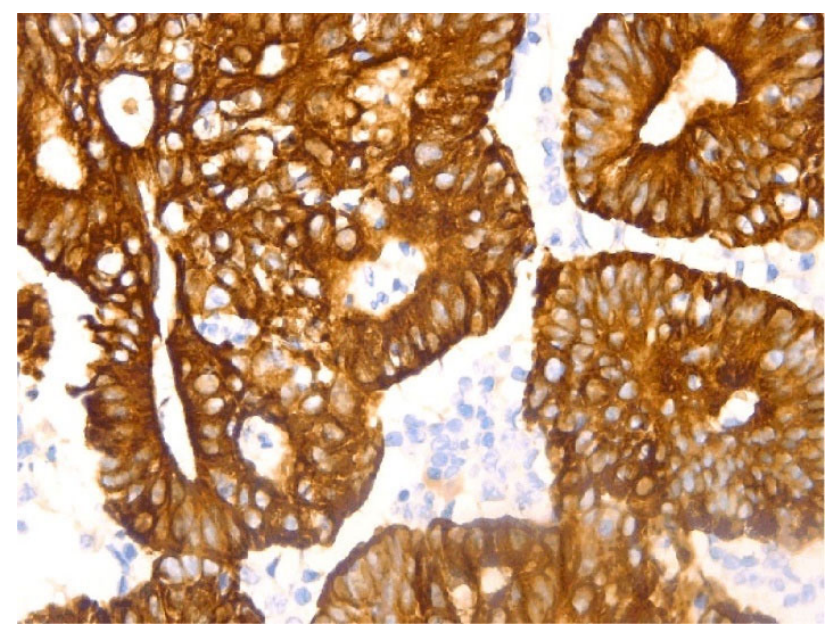

Figure 5 - Typical CK7 membranous and cytoplasmic immunopositivity of endometrial adenocarcinoma. AntiCK7 antibody immunostaining, $\times 400$. CK7: Cytokeratin 7.

Some other circumstances in which an endometrial neoplasia could be discovered without clinical manifestation is following the endometrial thickness by transvaginal US examination. Many studies, including that conducted by Timmermans et al., recommend transvaginal US examination as a screening for endometrial cancer, using a cut-off size $3 \mathrm{~mm}$ over which endometrial sampling is indicated [6].

Endometrial biopsy has a good sensitivity $(90 \%)$ in detecting endometrial cancer but only if the biopsy is hysteroscopic directed. Blind biopsy less reliable has more false negative results and is more invasive [7]. In our case, biopsy was not indicated and had no benefits. condition. In our Clinic, in a 5-year period, we had 846 hysterectomies for benign condition and only five $(0.59 \%)$ incidental uterine neoplasia, which is far below the literature data [5]. All cases were in women over 55 years old, but no other case was found on the tip of a type 0 myoma.

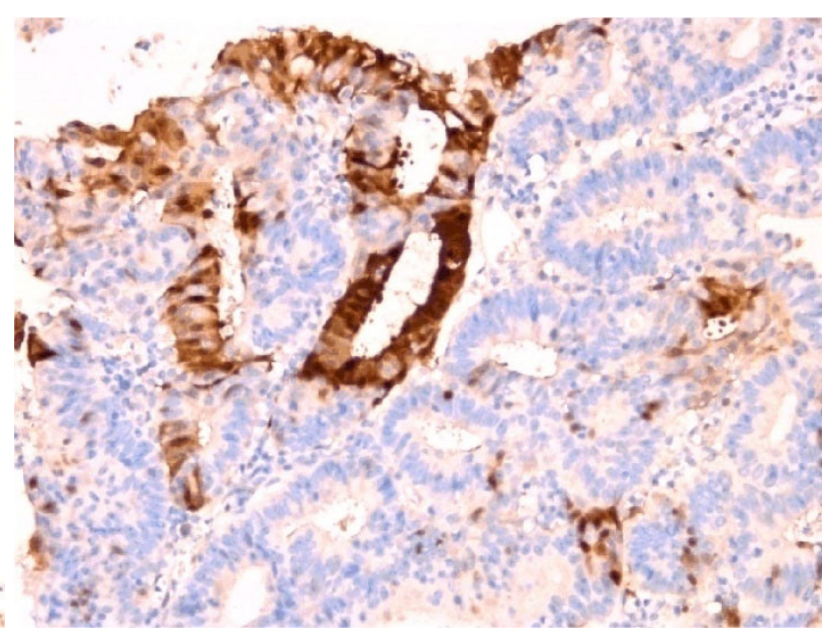

Figure 4 - A p16 mosaic, patchy positive mostly cytoplasmic reaction was found in the area of interest. Anti-p16 antibody immunostaining, $\times 200$.

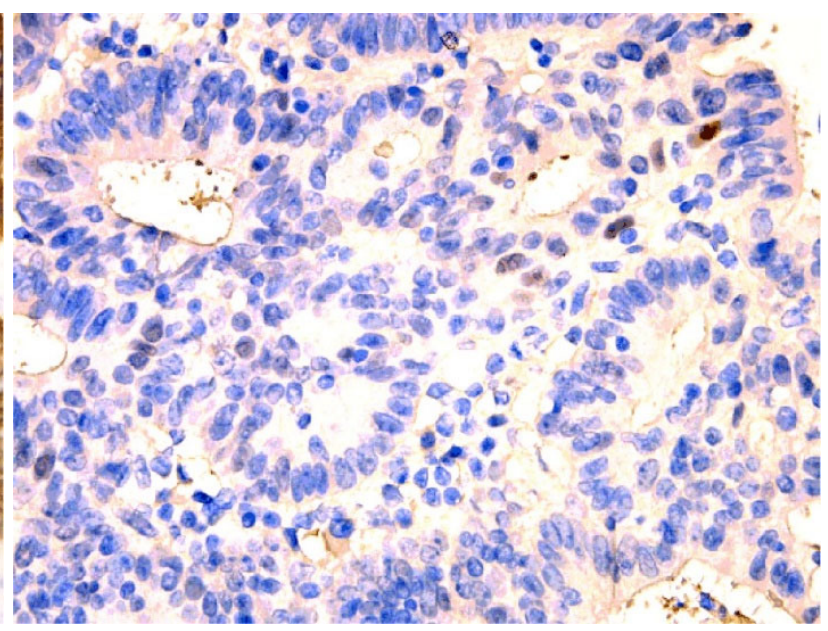

Figure 6 - p53 weak expression in scattered nuclei, defining a TP53 normal reaction. Anti-p53 antibody immunostaining, ×400. TP53: Tumor protein 53.

Random punch biopsy of the myoma was not an option and had the risk of missing the neoplastic areas due to the necrosis. Moreover, endometrial sampling could not be safely performed and had also no benefit considering the reduced endometrial thickness proved by transvaginal US scan.

Endometrioid ADK accounts for $80 \%$ of the endometrial carcinoma can be divided in two categories, according to ERs, and thus are responding different to antiestrogen therapy [8]. In our case, the important percent of ERs put the case in the estrogen-sensitive type. That fact and the unusually growing myoma in period when estrogens are normally at a very low level risen the hypothesis of 
the non-physiological secretion of estrone in fatty tissue as a common etiology (obese patient). Moreover, obesity seems to intervene in endometrial neoplasia in association with the chronic inflammation syndrome and hyperglycemia that fuels the neoplastic changes in endometrial cells [9]. Considering the associated comorbidities, our patient had those risk factors that could favor the neoplastic process but in an odd manner, the changes took place in an atypical spot covering the myoma and not on the heterotopic endometrium.

Well-differentiated endometrioid ADK has a glandular structure similar to the normal endometrium and can be associated or preceded by endometrial hyperplasia. According to Conlon et al., the ratio of glandular structure ( $<5 \%$ for grade 1 and $>50 \%$ for grade 3 ) divides into the three degrees of histological differentiation [10]. In our case, there were no simultaneous hyperplasic lesions at the level of the neoplasia and the heterotopic endometrium was atrophic, excluding the hypothesis of preneoplastic lesion.

IHC tests [ERs, progesterone receptors (PR), p53, p16, $\mathrm{Ki67}$, phosphatase and tensin homolog (PTEN)] are useful considering that there are some uncertain morphological ADKs of uncertain or hybrid type that need to be correctly labeled. Such types are serous carcinoma characterized by $p 53$ gene mutation and chromosomal instability and can associate a serous intraepithelial carcinoma (intraepithelial endometrial carcinoma), considered as a precursor lesion [11]. Also, in some cases, gynecological tumors may be in fact metastases of other occult cancers or may have unusual metastases and IHC tests may help in establishing the origin and histological subtype of the primary neoplasia $[4,12]$. Depending on the histological subtype, gynecological tumors can respond differently to therapy, so a tailored management plan is always preferred [13]. In our case, the presence of positive ERs and the low Ki67 index (10-15\%) indicates a low malignancy index, a low risk of lymphatic or vascular emboli and a good prognosis, according to the literature data [14].

Considering the clinical and paraclinical data, the case was staged as IA G1, low risk for relapse and metastasis and managed according to the recommendation by simple hysterectomy and adnexectomy. No benefits were shown to extend the procedure and perform pelvic or paraaortic lymphadenectomy in such stages [15]. In our case, since the malignancy was not suspected, to perform a new procedure in order to explore the pelvic nodes was not necessary and was endorsed by MRI examination that did not identify any metastatic sites or lymph nodes three months after the initial procedure.

\section{a Conclusions}

We present a peculiar endometrial ADK case that was accidentally discovered in an unusual specimen (a necrotized type 0 myoma) without any other symptom. The patient had risk factors for ADK (obesity and type II diabetes) but strangely the heterotopic endometrium did not react at the high levels of estrogens. Usually, ADKs can be found on endometrial polyps and sometimes are concomitant with myomas in premenopausal women but there are no reports in the literature about ADK developed on myomas in menopause. The most interesting aspect of the case was the unusual presence of an active myoma in menopause and the totally unsuspicious US appearance of the endometrium, which was below the cut-off size for biopsy indication and the poor Doppler supply suggesting only the submucosal necrotic myoma. This case is highlighting the ADK should always be considered, and cautious examination should be performed in menopausal women presenting with uterine tumors and or bleeding, even if the first impression is one of a benign condition. This case highlights the importance of considering the differential diagnosis of uterine bleeding and the need for further investigation in selected cases.

\section{Conflict of interests}

The authors declare that they have no conflict of interests.

\section{References}

[1] World Health Organization (WHO). GLOBOCAN - Global Cancer Observatory (GCO). International Agency for Research on Cancer (IARC), 12 July 2013. http://globocan.iarc.fr

[2] National Cancer Institute (NCl). Epidemiology of Endometrial Cancer Consortium (E2C2). Division of Cancer Control \& Population Sciences, Epidemiology and Genomics Research Program, accessed: October 10, 2019. https://epi.grants. cancer.gov/eeccl

[3] Zeleniuch-Jacquotte A, Akhmedkhanov A, Kato I, Koenig KL, Shore RE, Kim MY, Levitz M, Mittal KR, Raju U, Banerjee S, Toniolo P. Postmenopausal endogenous oestrogens and risk of endometrial cancer: results of a prospective study. $\mathrm{Br} \mathrm{J}$ Cancer, 2001, 84(7):975-981. https://doi.org/10.1054/bjoc. 2001.1704 PMID: 11286480 PMCID: PMC2363831

[4] Neaç̧u A, Marcu ML, Stănică CD, Brăila AD, Pacu I, loan RG, Grigorescu CC, lonescu CA. Clinical and morphological correlations in early diagnosis of endometrial cancer. Rom J Morphol Embryol, 2018, 59(2):527-531. PMID: 30173258

[5] Desai VB, Wright JD, Gross CP, Lin H, Boscoe FP, Hutchison LM, Schwartz PE, Xu X. Prevalence, characteristics, and risk factors of occult uterine cancer in presumed benign hysterectomy. Am J Obstet Gynecol, 2019, 221(1):39.e1-39.e14. https://doi. org/10.1016/j.ajog.2019.02.051 PMID: 30853364 PMCID: PMC7006101

[6] Timmermans A, Opmeer BC, Khan KS, Bachmann LM, Epstein E, Clark TJ, Gupta JK, Bakour SH, van den Bosch T, van Doorn HC, Cameron ST, Giusa MG, Dessole S, Dijkhuizen FPHLJ, Ter Riet G, Mol BWJ. Endometrial thickness measurement for detecting endometrial cancer in women with postmenopausal bleeding: a systematic review and metaanalysis. Obstet Gynecol, 2010, 116(1):160-167. https://doi. org/10.1097/AOG.0b013e3181e3e7e8 PMID: 20567183

[7] Torres ML, Weaver AL, Kumar S, Uccella S, Famuyide AO, Cliby WA, Dowdy SC, Gostout BS, Mariani A. Risk factors for developing endometrial cancer after benign endometrial sampling. Obstet Gynecol, 2012, 120(5):998-1004. https:// doi.org/10.1097/aog.0b013e31826b9fef PMID: 23090515 PMCID: PMC3711271

[8] Kurman RJ, Carcangiu ML, Herrington CS, Young RH (eds). World Health Organization (WHO) Classification of tumours of the female reproductive organs. $4^{\text {th }}$ edition, International Agency for Research on Cancer (IARC) Press, Lyon, France, 2014, 126, 150.

[9] Onstad MA, Schmandt RE, Lu KH. Addressing the role of obesity in endometrial cancer risk, prevention, and treatment. $\mathrm{J}$ Clin Oncol, 2016, 34(35):4225-4230. https://doi.org/10. 1200/JCO.2016.69.4638 PMID: 27903150 PMCID: PMC 5455320

[10] Conlon N, Leitao MM Jr, Abu-Rustum NR, Soslow RA. Grading uterine endometrioid carcinoma: a proposal that binary is best. Am J Surg Pathol, 2014, 38(12):1583-1587. https://doi.org/ 10.1097/PAS.0000000000000327 PMID: 25229772 
[11] Fadare O, Liang SX, Ulukus EC, Chambers SK, Zheng W. Precursors of endometrial clear cell carcinoma. Am J Surg Pathol, 2006, 30(12):1519-1530. https://doi.org/10.1097/01. pas.0000213296.88778.db PMID: 17122507

[12] Herghelegiu CG, Neacşu A, Oprescu ND, Cărbunaru AE, Brăila $A D$, Curea FG, Marcu ML, loan RG, Bohîlţea RE. Difficulties of clinical and histopathological diagnosis in advanced vulvar clear cell carcinoma. Rom J Morphol Embryol, 2018, 59(4):1233-1237. PMID: 30845306

[13] Botnariuc I, llie SM, Trifanescu OG, Bacinschi XE, Curea F, Anghel RM. Predictive circulating markers for anthracycline chemotherapy in non-metastatic breast cancer. Acta Endocrinol (Bucharest), 2017, 13(2):209-214. https://doi.org/10.4183/aeb. 2017.209 PMID: 31149175 PMCID: PMC6516453

[14] Salama A, Arafa M, EIZahaf E, Shebl AM, Awad AAE, Ashamallah SA, Hemida R, Gamal A, Foda AA, Zalata K,
Abdel-Hady EM. Potential role for a panel of immunohistochemical markers in the management of endometrial carcinoma. J Pathol Transl Med, 2019, 53(3):164-172. https://doi.org/ 10.4132/jptm.2019.02.12 PMID: 30813708 PMCID: PMC 6527935

[15] Benedetti Panici P, Basile S, Maneschi F, Alberto Lissoni A, Signorelli M, Scambia G, Angioli R, Tateo S, Mangili G, Katsaros D, Garozzo G, Campagnutta E, Donadello N, Greggi S, Melpignano M, Raspagliesi F, Ragni N, Cormio G, Grassi R, Franchi M, Giannarelli D, Fossati R, Torri V, Amoroso $M$, Crocè $C$, Mangioni $C$. Systematic pelvic lymphadenectomy vs. no lymphadenectomy in early-stage endometrial carcinoma: randomized clinical trial. J Natl Cancer Inst, 2008, 100(23):1707-1716. https://doi.org/10.1093/jnci/djn397 PMID: 19033573

\section{Corresponding author}

Romina-Marina Sima, Assistant Professor, MD, PhD, Department of Obstetrics and Gynecology, Carol Davila University of Medicine and Pharmacy, Bucharest; Department of Obstetrics and Gynecology, Bucur Maternity, St. John Clinical Hospital, 10 Între Gârle Street, Sector 4, 040294 Bucharest, Romania; Phone +40741-071 243, e-mail: romina.sima@yahoo.es

Received: February 19, 2020

Accepted: March 18, 2021 